\title{
An integrated pharmacokinetic and imaging evaluation of vehicle effects on solute human epidermal flux and, retention characteristics
}

G Winckle $^{1,3}$, YG Anissimov ${ }^{1,4}$, SE Cross ${ }^{1}$, G Wise $^{2,5}$ and MS Roberts ${ }^{1 \text { * }}$

1 Therapeutics Research Unit, Southern Clinical School, University of Queensland, Princess Alexandra Hospital, Brisbane, Queensland 4102, Australia, 2 School of Biomedical Sciences, University of Queensland, Brisbane, Queensland 4072, Australia,

Present address: 3 Dow Pharmaceutical Sciences, Inc., Petaluma, California 94954, United States of America, 4 School of Biomolecular and Physical Sciences, Griffith University, Nathan, Queensland 4111, Australia, 5 Institute for Glycomics, Griffith University (Gold Coast Campus), Queensland 9726, Australia

*To whom correspondence should be addressed. Phone +6173240 2546, Fax +61 73240 5806, email: m.roberts@uq.edu.au

Keywords: Transdermal penetration, stratum corneum, multiphoton laser scanning microscopy, formulation, corneocyte. 


\begin{abstract}
Our understanding of the differential effects of topically applied vehicles on solute partitioning and diffusion within the skin is presently limited. In this work, in vitro epidermal partitioning, penetration and multiphoton laser scanning microscopy (MPLSM) imaging studies were used to assess the distribution of 2-naphthol across human epidermis. Four commonly used liquid vehicles (100\% water, $20 \%$ propylene glycol (PG)/water, 50\% ethanol (EtOH)/water and 100\% isopropyl myristate (IPM)) were used. The maximum flux and membrane retention of 2-naphthol from $50 \%$ $\mathrm{EtOH} /$ water was almost an order of magnitude or larger than from the other vehicles evaluated whereas IPM resulted in the highest membrane retention and lowest membrane penetration for 2-naphthol than other vehicles. MPLSM studies showed that 2-naphthol solute partitioned favourably into the intercellular lipids and that there was a vehicle-dependent uptake of 2-naphthol into corneocytes. This study, using an integrated evaluation using in vitro penetration, epidermal retention and MPLSM imaging, has shown that vehicle effects on skin penetration include an alteration in the distribution of solutes between the corneocytes and intercellular lipids as well as by the well known mechanisms of altered partitioning into the stratum corneum and enhanced epidermal solute pathlength diffusivity.
\end{abstract}




\section{Introduction}

The skin is a structurally complex and heterogeneous membrane (1), which has a number of functions including retention of body water and the prevention/limitation of systemic absorption of xenobiotics following topical exposure. The stratum corneum (SC) is widely considered as the main barrier to transdermal penetration (2). Three SC transport routes have been postulated: transcellular, intercellular and transappendageal (2). However, the relative contribution of each pathway and the effects of different vehicles on each route have yet to be fully elucidated.

Evidence exists for transport through and between cells and via follicles and associated appendages. Historically, trancellular transport was initially thought to be dominant (2). However, the large diffusional resistance offered by multiple hydrophilic/hydrophobic partitioning steps, the cornified envelope, covalently bound lipid envelope and the compact keratin/filaggrin matrix has resulted in this pathway being largely discounted as a significant contribution to SC penetration. Current evidence, however. supports some degree of uptake of certain compounds into corneocytes, including the penetration of mercuric chloride particles into apical corneocytes (3), corneocyte uptake of fluorescent probes, propylene glycol interactions with corneocyte envelope proteins and/or keratin fibres (4) and corneocyte swelling mediated by water uptake (5).

One present prevailing view is that transport occurs through the intercellular lipids between corneocytes (6). Evidence supporting intercellular transport includes the inverse linear relationship between percutaneous absorption of benzoic acid and corneocyte diameter (7), although the permeability of benzoic acid in this work can also be argued to vary inversely with SC thickness and as such provides only weak evidence for intercellular permeation.. Furthermore, increased water flux (9) and transepidermal water loss (TEWL) (10) following SC delipidisation indicate the importance of these lipids in providing a barrier resistance to water. Recently, however, Wang et al suggested transcorneocyte transport may be more prevalent for a wider range of solutes than previously thought (11). Several compounds have also been localised in the skin appendages and follicular targeting is becoming 
increasingly important for micro-particulate (12), vesicular (13) iontophoretic (14) a and macromolecular (15) solute delivery.

Skin penetration and uptake is often assessed using in vitro human or animal skin in static or flow through cells. Using visual techniques to understand skin transport mechanisms is less common. Significant penetration studies involving imaging have utilised electron $(3,16,17)$ and confocal (18-24) microscopy. A recent visualisation technique applied to skin penetration is multiphoton laser scanning microscopy (MPLSM). The advantages of using MPLSM to examine biological tissues such as skin membranes have been previously described (25-28). Principal advantages of MPLSM over conventional confocal fluorescence microscopy include reduced light scattering and reduced tissue damage. In relatively thick, opaque samples such as epidermal membranes, these factors can improve sample penetration, axial resolution and signal to noise ratio. This is essential when dealing with highly light scattering and morphologically heterogeneous samples such as human heat separated epidermal membranes. Furthermore, this technique enables the visualisation of UV excitable compounds.

In this study, classical penetration/partitioning studies were combined with MPLSM imaging to determine how commonly used cosmetic/pharmaceutical vehicles affect the transport mechanism of 2-naphthol across human epidermal membranes. The relative differences in the uptake and transport of 2-naphthol in the intercellular lipids and corneocytes of the SC due to vehicle-skin interactions was of particular interest.

\section{Materials and Methods}

\section{Materials}

2-Naphthol (99\%), 2-naphthol-8- ${ }^{14} \mathrm{C}(99.1 \% ; 4.7 \mathrm{mCi} / \mathrm{mmol})$, isopropyl myristate (IPM, 98\%), bovine serum albumin (BSA, 98\%) and phosphate buffered saline $(\mathrm{pH}$ 7.4, PBS) sachets were purchased from Sigma-Aldrich (Castle Hill, New South Wales, Australia). HPLC grade acetonitrile and acetic acid and AR grade ethanol $(\mathrm{EtOH})$ and propylene glycol (PG) were obtained from Sigma-Aldrich. Ultima Gold scintillant and Soluene 350 tissue solubilizer were purchased from Perkin Elmer Life 
Sciences (Rowville, Victoria, Australia). Disposable base moulds and Cryomatrix, frozen section embedding resin, were purchased from ThermoTrace (Noble Park, Victoria, Australia).

\section{Human skin preparation}

Female abdominal skin was obtained from one Caucasian donor (52 years) following abdominoplasty. The subcutaneous fat was removed by blunt dissection and epidermal membranes were prepared by heat separation (29). All membranes were prepared on the same day as excision, cut into disks using a cork borer and frozen at $-20^{\circ} \mathrm{C}$ until use. Membranes were used within 4 months of excision and preparation.

\section{Solubility determination}

Excess solid 2-naphthol was added to $1.5 \mathrm{ml}$ of each vehicle $(100 \%$ water, $20 \% \mathrm{v} / \mathrm{v}$ $\mathrm{PG} /$ water, $50 \% \mathrm{v} / \mathrm{v} \mathrm{EtOH} /$ water and 100\% IPM, $\mathrm{n}=5)$. The samples were continuously agitated by magnetic stirrer bars and incubated in a water bath at $32^{\circ} \mathrm{C}$ for 72 hours. Samples were subsequently centrifuged at 10000 rpm for 10 minutes at $32^{\circ} \mathrm{C}$. The supernatant was removed, diluted with acetonitrile and assayed for 2naphthol by HPLC. In addition, the solubility of 2-naphthol in $4 \% \mathrm{w} / \mathrm{v}$ BSA/PBS (receptor phase) was also determined by the same method as described above but with an additional protein precipitation step involving the addition of $2: 1$ ratio of acetonitrile (containing $100 \mathrm{mcg} / \mathrm{ml}$ phenol as an internal standard) to each sample.

\section{Donor phases}

All donor phases were saturated with excess 2-naphthol, ensuring that precipitate was present. The 100\% water, 20\% PG/water and 50\% EtOH vehicles were prepared as saturated solutions containing both cold (unlabelled) and radiolabelled $\left[{ }^{14} \mathrm{C}\right]$ 2-naphthol. However, due to the high solubility of 2-naphthol in IPM and low epidermal penetration observed from this vehicle, only cold permeant was used to produce the saturated donor phase and all samples were assayed by HPLC. Donor phases containing cold and radiolabelled permeant were prepared by adding sufficient radiolabel to produce hot/cold mixtures of at least $10000 \mathrm{dpm} / \mathrm{mg}$ of cold 
permeant. Radiolabel was added to an accurately weighed amount of cold permeant using a glass $250 \mu \mathrm{l}$ glass syringe (Hamilton Co., Reno, Nevada, USA). Subsequently, $20 \mathrm{ml}$ of EtOH was added and the contents were thoroughly agitated for 5 minutes by vortex mixing. The $\mathrm{dpm} / \mathrm{mg}$ of the hot/cold mixtures was determined by removing $5 \times 10 \mu$ samples and assaying by liquid scintillation counting (LSC). Ethanol was removed under a stream of nitrogen overnight and the remaining solid was subsequently re-suspended in the appropriate vehicle.

\section{Penetration and distribution studies}

Human heat separated epidermal membranes (HHSEM) were placed on a filter paper support and mounted in side-by-side glass diffusion cells (supplied by the University of Queensland glass blowing services, diffusional surface area $0.76 \pm 0.07$ $\mathrm{cm}^{2}$, volume $3.66 \pm 0.15 \mathrm{ml}$, mean $\pm \mathrm{SD}$ ). These were positioned in a Perspex support on a submersible stirrer plate and placed in a stirred temperature controlled water bath at $32 \pm 0.1^{\circ} \mathrm{C}$. Membrane integrity was determined using conductivity measurements following a 1 hour equilibration period (only skin samples with conductivity less than $50 \mu \mathrm{Si} \mathrm{cm}^{-2}$ were used). As PBS was used as the conducting medium, the donor and receptor compartments were thoroughly rinsed with either blank vehicle (no permeant present) or receptor phase, respectively. A 4\%BSA/PBS solution was employed as the receptor phase and was agitated by magnetic stirrer bars. The experiment duration was 4 hours and $200 \mu$ l samples were removed from the receptor compartment at 15,30,60, 120, 180 and 240 minutes (6-9 replicates were used for each vehicle). In all cases, the concentration of 2-naphthol in the receptor phase remained below $10 \%$ of the saturated solubility in the receptor phase (30) which was measured to be $2.94 \pm 0.08 \mathrm{mg} / \mathrm{ml}$. After 4 hours, the cells were dismantled and the diffusional area was wiped with a tissue and excised using a cork borer. The excised diffusional area was then wiped with a cotton bud, which had been dipped in 50\% v/v aqueous $\mathrm{EtOH}$, to remove any residual 2-naphthol. Two samples were retained for microscopic evaluation while the 2-naphthol content of the remainder was quantified. $200 \mu \mathrm{l}$ of each receptor phase sample containing radiolabelled $\left[{ }^{14} \mathrm{C}\right] 2$-naphthol was added to $2 \mathrm{ml}$ of scintillant and vortexed for 30 seconds. Subsequently samples were counted for 2 minutes on a Packard (Canberra, Australia) Tri-carb TR 2700 liquid scintillation counter whilst those samples containing unlabelled 2-naphthol only were quantified by HPLC. 
Membrane content analysis

The 2-naphthol content of the diffusional area of the epidermal membranes exposed to the $100 \%$ water, $20 \% \mathrm{v} / \mathrm{v} \mathrm{PG} /$ water and $50 \% \mathrm{v} / \mathrm{v} \mathrm{EtOH} /$ water vehicles was determined by LSC. The excised diffusional areas were placed in $1 \mathrm{ml}$ of Soluene 350 tissue solubilizer (PerkinElmer Life Sciences, Victoria, Australia) at room temperature and were agitated using a gyratory rocker. After 24 hours, $200 \mu$ samples were removed and added to $2 \mathrm{ml}$ of Ultima Gold scintillant (PerkinElmer Life Sciences, Victoria, Australia) and vortexed. All samples were light adapted for at least 1 hour, to ensure all chemiluminescence had subsided, prior to counting (31).

Epidermal samples that were exposed to the IPM vehicle were extracted with acetonitrile $(1 \mathrm{ml})$ containing phenol $(10 \mu \mathrm{g} / \mathrm{ml})$ as an internal standard. Samples were incubated in the extraction medium for 24 hours at room temperature and agitated using a gyratory rocker. Subsequently, $200 \mu \mathrm{l}$ samples of the extraction medium were subjected to HPLC analysis. The data obtained using acetonitrile extraction agreed closely with preliminary results acquired using radiolabelled techniques.

\section{HPLC Analysis}

HPLC was performed using a Shimadzu Scientific Instruments isocratic system (Rydalmere, New South Wales, Australia) with a Phenomenex $5 \mu \mathrm{m}$ Luna ODS column $(4.6 \mathrm{~mm} \times 250 \mathrm{~mm}$, coupled to a Phenomenex guard cartridge system). The mobile phase consisted of $55 \%$ acetonitrile, $44 \%$ water and $1 \%$ glacial acetic acid and was delivered at a flow rate of $1 \mathrm{ml} / \mathrm{min}$ by a LC-10AT pump. Samples were maintained at $20^{\circ} \mathrm{C}$ with a Shimadzu sample cooler rack $\left( \pm 0.1^{\circ} \mathrm{C}\right)$ and $10 \mu \mathrm{l} \mathrm{samples}$ were injected using a SIL-10A XL auto injector. UV absorbance of the analytes was measured at $270 \mathrm{~nm}$ with a model SPD-10AV UV-VIS detector. The HPLC system was controlled with a model SPA-10A $\mathrm{VP}$ controller and Class VP software (Shimadzu). Under these conditions the run time was 5 minutes and the retention times of phenol and 2-naphthol were 2.7 and 4.1 minutes respectively. The limits of quantification were $50 \mathrm{ng} / \mathrm{ml}$ for phenol and $25 \mathrm{ng} / \mathrm{ml}$ for 2-naphthol. In all instances calibration standards were treated in the same manner as the unknown samples. 


\section{Data analysis}

When SC is the main barrier, the maximum flux of a solute $\left(J_{\max }\right)$ can be determined as follows [Roberts, 2002 \#246]:

$$
J_{\max }=\frac{S_{s c} D_{e f f}}{l}
$$

where $S_{s c}$ is the solubility of the solute in the vehicle, $D_{\text {eff }}$ is the effective diffusion coefficient and $I$ is the membrane thickness. It should be emphasised that $S_{s c}, D_{\text {eff }}$ and $I$ are all potentially affected by the vehicle.

The permeability coefficient $\left(k_{p}\right)$ was determined from $J_{\max } / S_{v}$, where $S_{v}$ is the solubility in the vehicle. Estimations of the pathlength diffusivity (D), effectively a diffusion mass transfer coefficient, of 2-naphthol within the membrane were calculated from the concept of $J_{\max } / S_{s c}$, however the solubility of 2-naphthol in the membrane $\left(S_{s c}, \mu \mathrm{g} / \mathrm{ml}\right)$ was substituted by our available retention of solute in the membrane $\left(R_{m}, \mu \mathrm{g} / \mathrm{cm}^{2}\right)$ at the end of the study period (recognising that 2-naphthol was applied in vehicles as saturated solutions). The membrane thickness was assumed to be constant and $\mathrm{D}$, defined as $\mathrm{J}_{\max } / R_{m}$, had dimensions of $1 /$ time $(\mathrm{h})$, as used in previous publications $(32,33)$, and therefore can be thought of in terms of being a diffusion mass transfer rate.

Solute penetration through the epidermal barrier can be influenced by membrane/vehicle interactions such as modification of the solute solubility in the membrane and/or solute pathlength diffusivity within the membrane (kinetic effect). Alteration of solute pathlength diffusivity may be related to structural disruption of the SC resulting in reduced barrier function. Therefore, results were analysed using the approach of Cross et al. (32) and Rosado et al. (33) which enables the differentiation of vehicular effects on solute partitioning and pathlength diffusivity. This analysis suggests that, if the transport process and pathway of 2-naphthol in SC from each vehicles was identical, a linear relationship between observed $J_{\max }$ and $S_{s c}$ (as defined by $R_{m}$ ) would be expected from equation (1). However, changes in $J_{\max }$ that are not directly related to changes in $R_{m}$ arise from alterations in membrane pathlength diffusivity and therefore deviate from the line of proportionality shown in figure $3(32,33)$. 


\section{Statistical analysis}

Statistical analysis was carried out using Minitab 13 (Minitab Inc, PA, USA) and the non-normal distribution of the data necessitated non-parametric analysis using the Kruskal-Wallis test.

\section{Membrane preparation for microscopy}

The tissue samples retained for microscopic evaluation were prepared in one of two ways. Some membranes were cryo-sectioned while others were placed directly onto a microscope slide with the SC side facing up.

Membranes which were to be sectioned were placed in Cryomatrix mounting medium and frozen using dry ice, immediately after diffusion cell dismantling and surface wipe procedures. All samples were stored at $-70^{\circ} \mathrm{C}$ until just prior to the sectioning procedure. Sections (20 $\mu$ m thick) were cut perpendicular to the SC surface at $-20^{\circ} \mathrm{C}$ using a $0^{\circ}$ cutting angle on a Leica CM1850 cryostat (Leica Microsystems, Nussloch $\mathrm{GmbH}$, Nußloch, Germany). This procedure was used to minimise the potential effect of tissue/permeant translocation during sectioning. The sections were stored on slides and protected with cover-slips (secured by $3 \mathrm{M}$ tape) and maintained at $-70{ }^{\circ} \mathrm{C}$ until microscopic evaluation.

Unsectioned epidermal membranes were frozen using dry ice, secured with a cover slip and 3M adhesive tape immediately after diffusion cell dismantling and surface wipe procedures and maintained at $-70{ }^{\circ} \mathrm{C}$ until microscopic evaluation.

\section{Microscopy}

Multiphoton microscopy was carried out using a Bio-Rad Radiance 2000 MP multiphoton, coupled to a Coherent Scientific 5W Verdi/Mira tuneable Ti:Sapphire laser, and mounted on a Nikon TE300 inverted microscope using a Nikon S Fluor 40x / NA 1.30 objective lens. Peak multiphoton excitation of 2-naphthol was 
established at $800 \mathrm{~nm}$ by monitoring the peak emission of 2-naphthol when excited by a range of wavelengths between $720 \mathrm{~nm}$ and $910 \mathrm{~nm}$. 2-Naphthol fluorescence could be visualised using blue $(460 / 50 \mathrm{~nm})$, green $(515 / 30 \mathrm{~nm})$, and red (620/100 $\mathrm{nm}$ ) band pass emission filters, and was strongest using a red emission filter in combination with $800 \mathrm{~nm}$ excitation.

Immediately prior to image collection, samples were thawed and mounted in glycerol. All images and image stacks were collected using a non-descanned detector with and 630/100 band-pass emission filter. Sectioned samples were optically sectioned $10 \mu \mathrm{m}$ below the surface of the section to exclude any possible visualisation of tissue/permeant translocation artefacts created by microtome sectioning.

Image analysis

Semi-quantitative image analysis of fluorescence intensity in membranes was carried out by identifying and selecting membrane regions of interest and quantifying mean fluorescence intensity (MFI) using LaserPix (Bio-Rad Microscience Ltd, Hertfordshire, UK) image analysis software.

\section{Results}

Epidermal membrane penetration, maximum flux and permeability coefficients

The penetration of 2-naphthol through heat separated epidermal membranes following application as a saturated solution in the 4 vehicles over time is shown in figure 1. It is evident that steady state permeation of 2-naphthol is exists for each vehicle after $120 \mathrm{~min}$ and lag times, derived from steady state penetration, range from about 10 to $30 \mathrm{~min}$. The solubilities, fluxes and permeability coefficients derived fro 2-naphthol are shown in Table 1. 


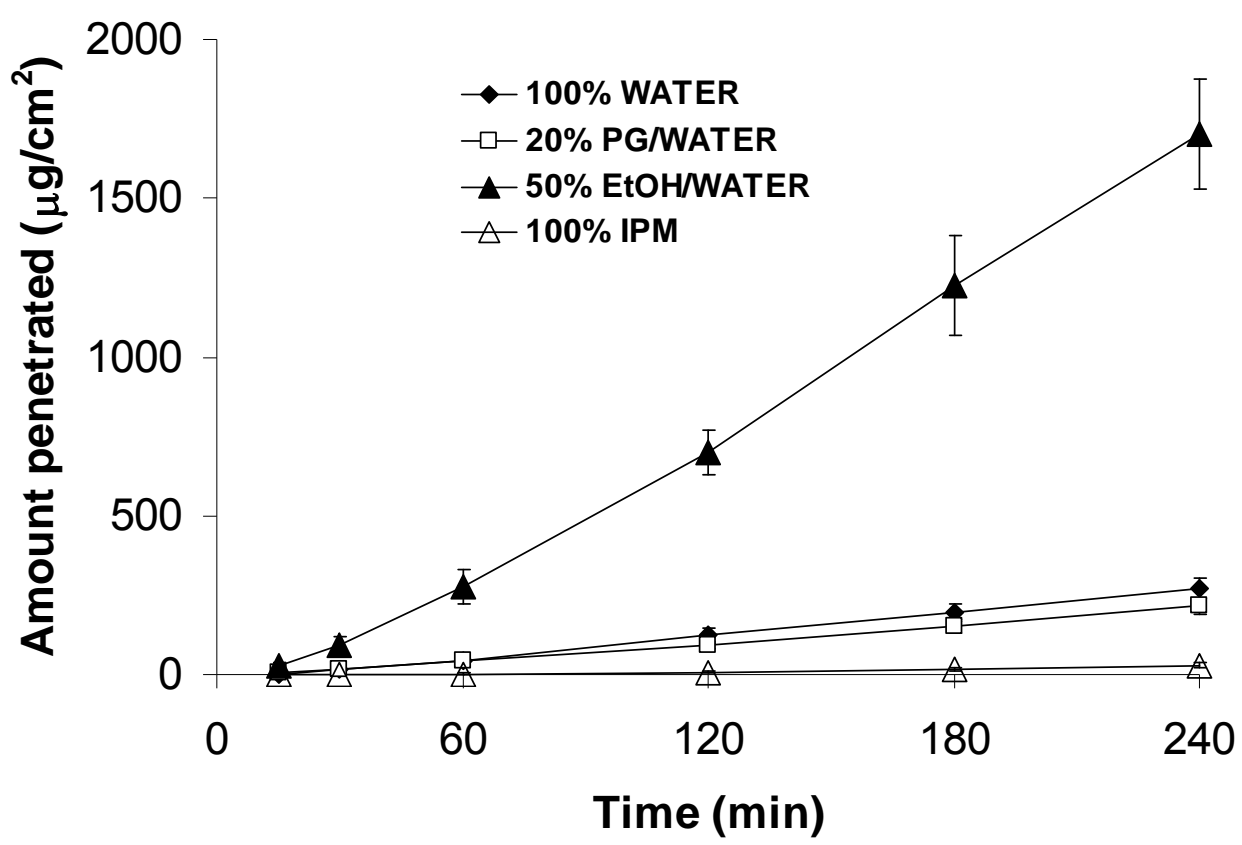

Figure 1: Cumulative amount of 2-naphthol penetrating HHSEM following application as saturated solutions in 4 vehicles: $100 \%$ water $(n=8), 20 \%$ PG/water $(n=8), 50 \% \mathrm{EtOH} /$ water $(n=9)$ and IPM $(n=6) ;($ mean $\pm S D)$.

Table 1: ${ }^{a}$ Saturated solubility of 2-naphthol in each vehicle and receptor phase at $32^{\circ} \mathrm{C}$.

${ }^{\text {b }}$ Maximum flux $\left(\mathrm{J}_{\max }\right)$ and permeability coefficients $\left(\mathrm{k}_{\mathrm{p}}\right)$ for saturated solutions of 2-naphthol applied to human heat separated epidermal membranes in different vehicles.

\begin{tabular}{l|cc|cc|cc}
\hline Formulation & \multicolumn{2}{|c|}{ Solubility $(\mathbf{m g} / \mathbf{m l})^{\mathbf{a}}$} & \multicolumn{2}{|c|}{$\mathbf{J}_{\max }\left(\mu \mathbf{g} / \mathbf{c m}^{2} / \mathbf{h}\right)^{\mathbf{b}}$} & \multicolumn{2}{|c|}{$\mathbf{k}_{\mathbf{p}} \times \mathbf{1 0}^{\mathbf{4}}(\mathbf{c m} / \mathbf{h})^{\mathbf{b}}$} \\
\hline & Mean & $\pm S D$ & Mean & $\pm S D$ & Mean & $\pm S D$ \\
\hline 100\% Water & 1.14 & 0.12 & 75 & 8.6 & 661 & 75 \\
$50 \%$ PG/Water & 1.90 & 0.25 & 57.3 & 6.6 & 301 & 34 \\
$100 \%$ IPM & 196 & 27 & 475 & 52 & 21.7 & 2.4 \\
$4 \%$ w/v BSA/PBS & 221 & 33 & 10.8 & 2.0 & 0.55 & 0.1 \\
\hline
\end{tabular}

it is apparent from Table 1 that the maximal flux $\left(J_{\max }\right)$ for 2-naphthol penetrating the epidermal membranes from 50\% EtOH/water was 6 fold or more greater than for $100 \%$ water, $20 \% \mathrm{PG} /$ water and $100 \%$ IPM vehicles $(p<0.004)$. The $100 \%$ water vehicle had a significantly higher $J_{\max }$ than the $20 \% \mathrm{PG} /$ water $(p=0.002)$ whereas the $100 \%$ IPM vehicle yielded a substantially lower $J_{\max }(p<0.004)$, approximately 7 and 5 times less than the $\mathrm{J}_{\max }$ for $100 \%$ water and $20 \%$ PG/water vehicles, respectively (Table 1).The permeability coefficients $\left(k_{p}\right.$, Table 1$)$ were reflective of the broad 
range of solubilities of 2-naphthol in the vehicles used. The $k_{p}$ of 2-naphthol was

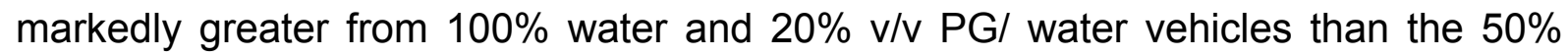
$\mathrm{EtOH} /$ water vehicle. The $100 \%$ IPM vehicle produced the lowest $k_{p}$. The $k_{p}$ for 2naphthol from the $100 \%$ water vehicle obtained in the present study $(0.066 \mathrm{~cm} / \mathrm{h})$ was in good agreement with literature values of 0.027 and $0.061 \mathrm{~cm} / \mathrm{h}$ as determined by Roberts et al. (34) and Hayden (35), respectively.

Membrane retention $\left(R_{m}\right)$

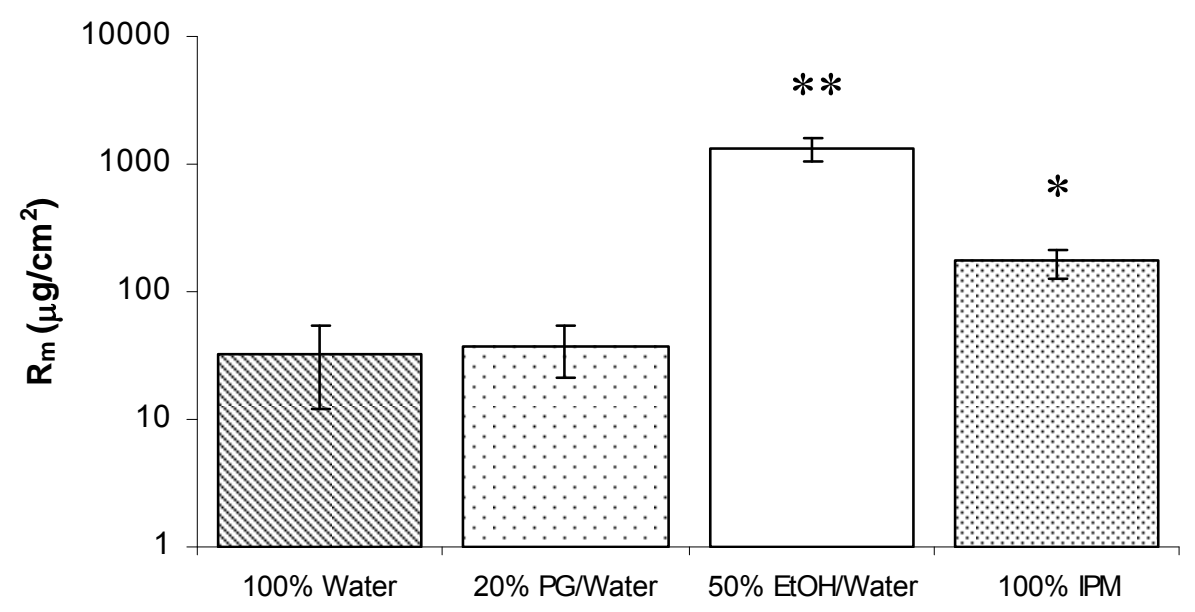

Figure 2: Membrane retention ( $R_{m}$, log scale) of 2-naphthol in HHSEM following application as saturated solutions of 4 vehicles: $100 \%$ water $(n=7), 20 \%$ PG/water $(n=7), 50 \% \mathrm{EtOH} /$ water $(n=7)$ and IPM $(n=6) ;($ mean $\pm S D, * *-p<0.005, *-p<0.004)$.

The amount of 2-naphthol retained in the membranes at the end of each study $\left(R_{m}\right)$ for eth 4 vehicles is shown in figure 2. The $50 \% \mathrm{EtOH} /$ water vehicle exhibited the highest 2-naphthol $R_{m}$ and was approximately 40, 35 and 8 times greater than the $100 \%$ water, $20 \%$ PG/water and IPM vehicles, respectively $(p<0.005)$. $R_{m}$ following application of 2-naphthol in 100\% IPM was approximately 4-5 times greater than from the $100 \%$ water and $20 \% \mathrm{PG} /$ water vehicles $(\mathrm{p}<0.004)$. No statistically significant difference was found between the membrane retention from the $100 \%$ water and $20 \%$ PG/water vehicles $(p=0.48)$. 


\section{Estimates of pathlength diffusivity}

The relationship between maximum flux $\left(J_{\max }\right)$ and membrane retention $\left(R_{m}\right)$ is shown in figure 3. An unchanged pathlength diffusivity for a given vehicle, relative to the $100 \%$ water vehicle, corresponds to data points on the line of identity, whereas points below or above the line correspond to a reduced or increased pathlength diffusivity, respectively. It is evident from fig. 3 that the $50 \% \mathrm{EtOH} /$ water and $100 \%$ IPM vehicles are positioned below the line of proportionality, suggesting that they lead to a reduced diffusion and/or enhanced partitioning for 2-naphthol relative to the $100 \%$ water vehicle. The $20 \%$ PG/water vehicle was positioned only slightly below the line of identity for the $100 \%$ water vehicle.

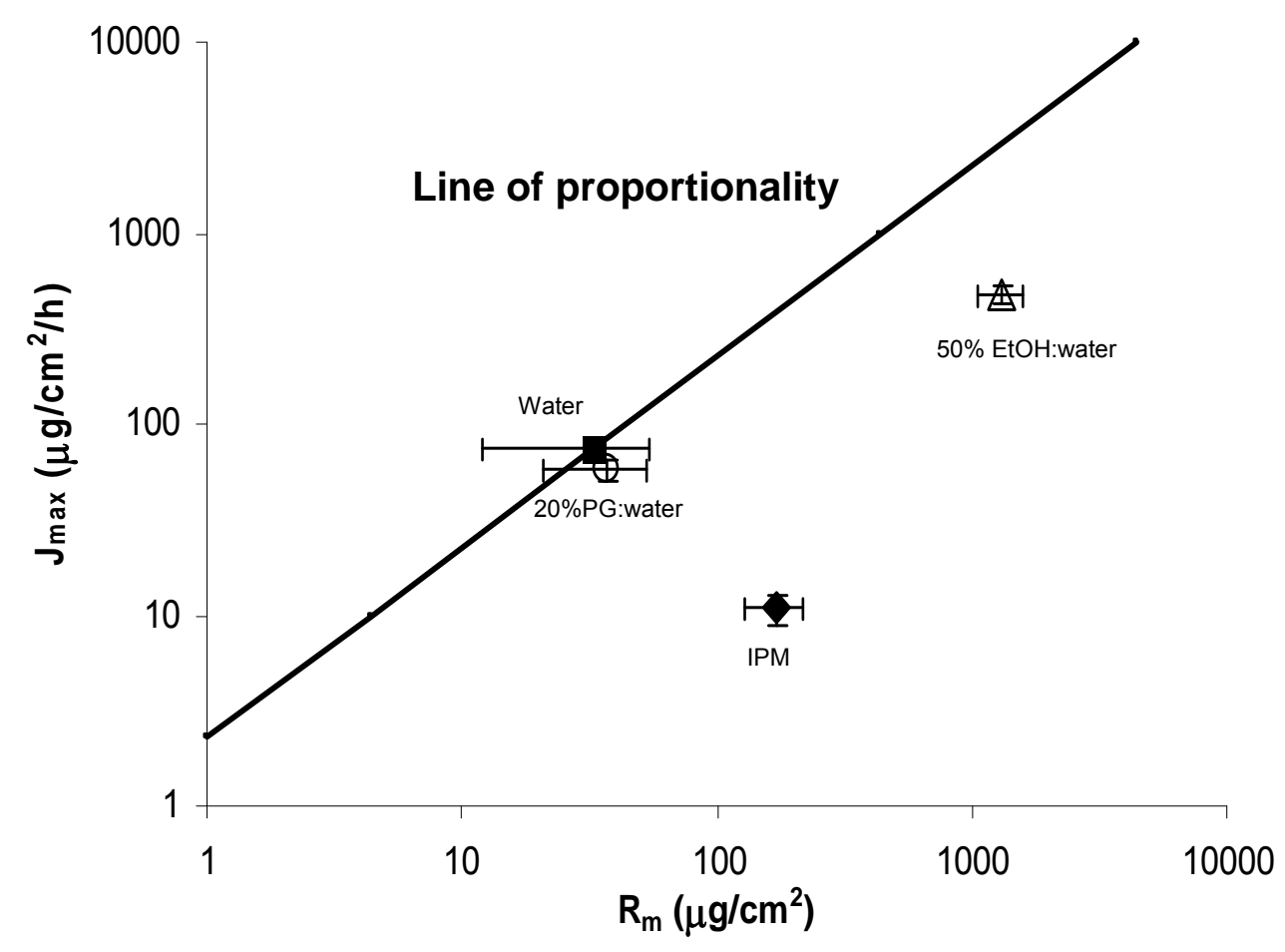

Figure 3: Relationship between maximum flux $\left(\mathrm{J}_{\max }\right)$ and membrane retention $\left(\mathrm{R}_{\mathrm{m}}\right)$ of 2-naphthol from $100 \%$ water, $20 \% \mathrm{PG} /$ water, $100 \% \mathrm{IPM}$ and $50 \% \mathrm{EtOH} /$ water vehicles (mean $\pm \mathrm{SD}$ ). The line of proportionality was plotted using the control vehicle, $100 \%$ water and all comparisons are discussed relative to water.

\section{Microscopy}

The fluorescence of 2-naphthol was detectable up to $60 \mu \mathrm{m}$ below the SC surface, in the axial-plane and was comparable, to the $80 \mu \mathrm{m}$ reported by Grewal et al. (36). Axial-plane sample penetration is dependent on the intensity of fluorescence 
emission, which is determined by probe concentration, probe extinction coefficient, light scattering by the skin and signal to noise ratio. Alternative compounds with larger extinction coefficients than 2-naphthol may facilitate visualisation deeper below the sample surface.

Cross sections (xs) of the human heat separated epidermal membranes exposed to each of the vehicles saturated with 2-naphthol and corresponding control samples (vehicle only) are shown in figures $4 \mathrm{~B}, \mathrm{C}, \mathrm{E}, \mathrm{F}, \mathrm{H}, \mathrm{I}, \mathrm{K}$ and $\mathrm{L}$. The membranes were examined in xs to evaluate the distribution of 2-naphthol fluorescence throughout the entire membrane. In all cases autofluorescence in control samples (Figure 4 C, F, I, L) was minimal compared with total fluorescence in 2-naphthol samples.

Figure 4: MPLSM images of human epidermis in plan view and cross section (xs). All scans were collected $10 \mu \mathrm{m}$ below the surface of each sample.

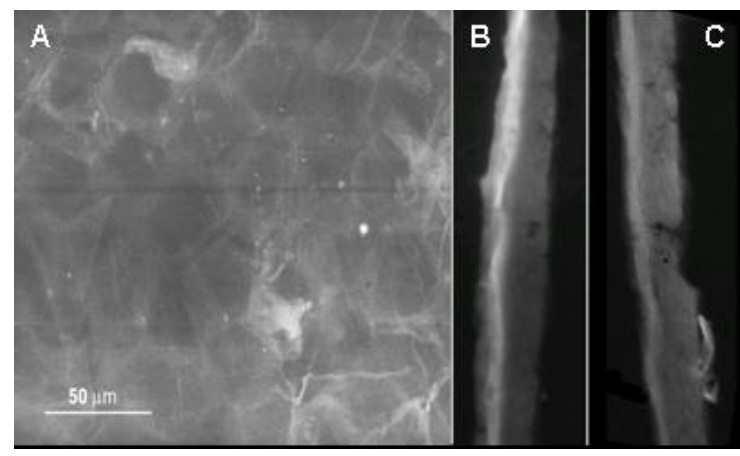

100\% water vehicle: A -Plan view; B sample cross section (XS); C - blank XS

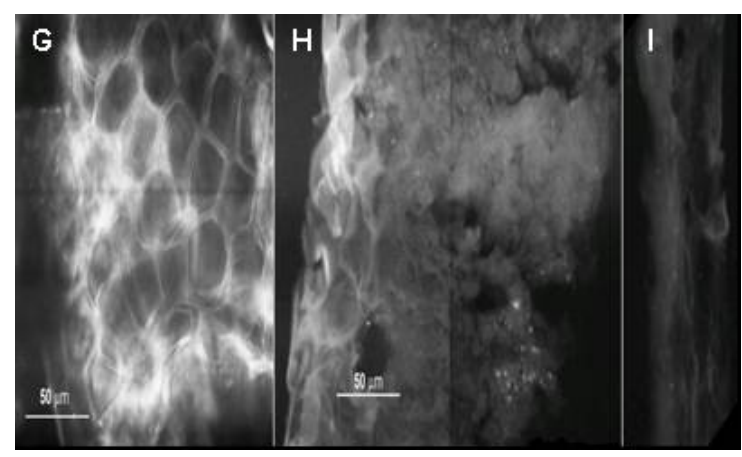

50\% EtOH/water vehicle: G -Plan view; $\mathrm{H}$ - sample XS; I - blank XS

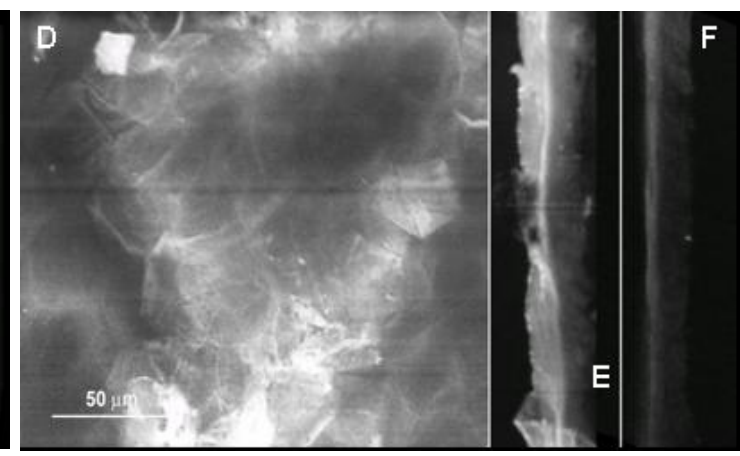

20\% PG/water vehicle: D -Plan view; E sample XS; F - blank XS

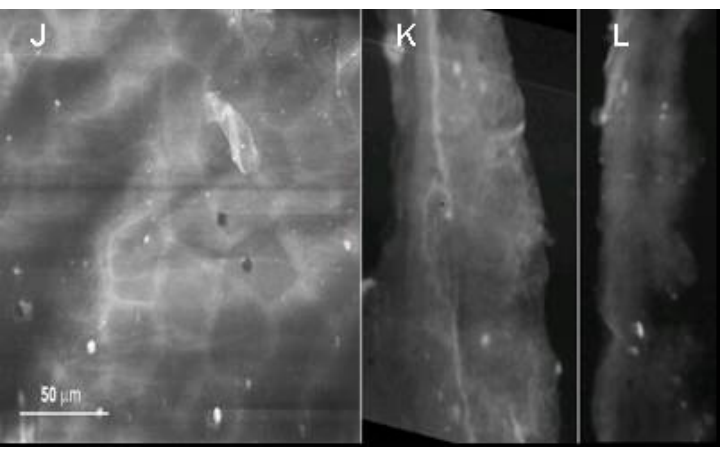

100\% IPM vehicle: J -Plan view; K sample XS; L - blank XS 
Table 2: MFI of 5 random samples from the intercellular region of the SC after 4 hour application of saturated solutions of 2-naphthol in 4 different vehicles. $\dagger$ - Calculated using $R_{m} / R_{m}$ water.

\begin{tabular}{l|cccc}
\hline Formulation & $\begin{array}{c}\text { Mean Fluorescence } \\
\text { Intensity(MFI) }\end{array}$ & $\pm S D$ & $\begin{array}{c}\text { MFI } \\
\text { vehicle:water } \\
\text { ratio }\end{array}$ & $\begin{array}{c}\text { Membrane retention }+ \\
\text { vehicle:water ratio }\end{array}$ \\
\hline $50 \%$ & 196.3 & 5.1 & 1.5 & 39.7 \\
EtOH/Water & 146.2 & 9.2 & 1.1 & 5.2 \\
$100 \%$ IPM & 129.1 & 1.5 & 1.0 & 1.0 \\
$100 \%$ Water & 112.0 & 6.5 & 0.9 & 1.1 \\
$20 \%$ PG/Water & & & &
\end{tabular}

Semi-quantitative image analysis of plan view images (Figure $4 \mathrm{~A}, \mathrm{D}, \mathrm{G}$ and J), using mean fluorescence intensity (MFI) (Table 2), indicated that the relative membrane retention of 2-naphthol from each vehicle (Figure 2) corresponded with the quantitative analysis illustrated in figure 2, with rank order $50 \% \mathrm{EtOH} /$ water $>100 \%$ IPM $>100 \%$ water $>20 \%$ PG/water in all cases. The different degrees of response between the MFI vehicle to water ratio and membrane retention vehicle to water ratio highlight the qualitative nature of the MFI analysis under the described conditions for 2-naphthol.

Figure $4 \mathrm{H}$ and $4 \mathrm{I}$ also indicate that the $50 \% \mathrm{EtOH} /$ water vehicle caused disruption of the epidermal morphology. These effects may be related to the higher $J_{\max }$ and $R_{m}$ exhibited by $50 \% \mathrm{EtOH} /$ water relative to the other vehicles evaluated. It was evident that the predominant localisation of 2-naphthol fluorescence is in the inter-corneocyte spaces; subsequent MFI samples were taken from these regions to facilitate comparisons. Control samples, exposed to the vehicle only (without 2-naphthol), were treated and visualised under identical conditions to the penetrant exposed membranes. Minimal autofluorescence was detected in plane view control samples viewed from SC surface (data not shown) and cross sectioned control samples (Figure 4 C,F,I,L)

\section{Discussion}

2-Naphthol was selected for this study based on having a range of desirable properties. These include its physicochemical properties for skin penetration $(M W=144.17, p k a=9.51, \log P=2.7$, Environmental Science Centre PhysProp online 
database experimental values), its fluorescent properties allowing visualisation using MPLSM (ex= 327nm, em=475nm) and its apparent non-damaging effect upon human skin compared with other phenolic compounds (34). 2-Naphthol has also been used as a topical antiseptic, counter irritant in alopecia and anthelmintic in humans and is therefore of therapeutic interest. The relatively low molecular weight $(37,38)$, moderate partition coefficient (38-40), low hydrogen bonding (41), and predominantly unionised state around physiological $\mathrm{pH}$ of 2-naphthol also make it a rapid skin penetrant, enabling skin penetration studies to be conducted over a relatively short time. Saturated solutions were used in this study assuring that the thermodynamic activity of the solute in each vehicle should be identical. Hence $J_{\max }$ should be identical, providing the vehicle does not alter the permeability properties of the SC (6). The use of saturated solutions also generated maximal tissue levels which facilitated the microscopic visualisation of the solute distribution in the SC.

\section{Limitations in current data analysis}

The current data analysis relied on eq 1 which is only valid if the membrane flux is controlled and retention is dominated by SC and not significantly influenced by VE. The fact that the permeability of the skin, and thus the flux, is determined mostly by $\mathrm{SC}$ for not too lipophilic solutes $(\log \mathrm{P}<3)$ is well established $(37)$ It follows therefore from two-layer membrane model that, at steady-state, the reduction in solute's activity will mainly occur in the SC, as the VE is exposed to concentrations of 2naphthol which are much less than that in the vehicle, and likely to be similar in activity to that in the receptor. Accordingly, the 2-naphthol concentration in VE at steady-state is estimated to be much less than its solubility in VE. If the solubility of 2naphthol in VE is similar in magnitude to that of $3 \mathrm{mg} / \mathrm{ml}$ for the $4 \%$ BSA receptor solution (Table 1) and the VE has a thickness of $100 \mu \mathrm{m}$, the estimated retention in the VE layer is $R_{V E}<30 \mu \mathrm{g} / \mathrm{cm}^{2}$. It is therefore concluded that the membrane retention measured $(\mathrm{Rm}>33 \mu \mathrm{g} / \mathrm{cm} 2$, for all vehicles, see figure 2$)$ is dominated by retention in SC.

\section{Proposed mechanisms of action of vehicles studied}

$\mathbf{5 0 \%} \mathrm{EtOH} /$ water vehicle. The 2-naphthol $J_{\max }$ from $50 \% \mathrm{EtOH} /$ water vehicle was enhanced about 6 times (Table 1) relative to water, consistent with the work of 
Berner et al (42), in which 5-10-fold increases in nitroglycerin flux from aqueous ethanol (50-70\%) were reported. The large increase in $R_{m}$ (almost 40 times, Figure 2) generated by the $50 \% \mathrm{EtOH} /$ water vehicle indicated a significant increase in partitioning into the membrane relative to water. However, it appeared that the pathlength diffusivity was reduced relative to $100 \%$ water vehicle as apparent from its position below the line of proportionality on Figure 3. The usual mechanism reported for $\mathrm{EtOH}$ enhancement of SC transport is through skin lipid and protein extraction and membrane disruption (43-45). This work suggests that an ethanol induced reduction in pathlength diffusivity may be synergistic with these mechanisms. Earlier studies have suggested that the diffusion rate of nitroglycerin remained unchanged in human SC from aqueous ethanol (50-70\%) (42) and that aqueous ethanol may lead to an extensive swelling of SC (46). However, it is emphasised that changes in pathlength diffusivity is only one contributor to overall transport as $R_{m}$ increases 40 times with $J_{\max }$ increasing 6 fold. It is also possible that a SC reservoir exists for the moderately lipophilic 2-naphthol and does not contribute directly to the observed flux. Further, the formation of isolated pools/lacunae containing the vehicle components within the SC following exposure to the vehicle $(16,47)$ may also contribute to such a reservoir. It has been suggested that $\mathrm{EtOH}$, and $\mathrm{PG}$, accumulate in the SC and enhance partitioning of a permeant or co-enhancer (48), which was consistent with the current experimental observations.

20\% PG/water vehicle. The use of a $20 \%$ PG solution in this study was consistent with its strength in commercial use. Formulations such as Rogaine $(2 \% \mathrm{w} / \mathrm{w}$ minoxidil) contain approximately $20 \% \mathrm{PG}$ in combination with $\mathrm{EtOH}$ and water (49). In addition, $20 \%$ PG/water solution has been recommended as a standard medium to evaluate contact dermatitis sensitivity in dermatological irritancy testing (50). A recent study also investigated a $20 \%$ PG/water vehicle and reported highly heterogeneous distribution of clobetasol propionate in human SC (51). In this work, the $J_{\max } 2$ naphthol from $20 \% \mathrm{PG} /$ water is only slightly reduced relative to the $100 \%$ water vehicle (Table 1, Fig 1) with a slight increase in membrane retention ( Fig. 3, Tbale 2). As a consequence, only a slight decrease in pathlength diffusivity is apparent (figure 2). 
$100 \%$ IPM vehicle. The current data shows that IPM increased the partitioning, and therefore membrane uptake of 2-naphthol, whilst also significantly decreasing the pathlength diffusivity in the SC (Figure 3). Permeation of IPM into the SC has been demonstrated $(52,53)$ and has been suggested to decrease SC lipid polarity (54). Furthermore, Brinkmann and Muller-Goymann (52) have observed increased lipid bilayer packing density and disorder of the covalently linked corneocyte-lipid envelope following IPM uptake by the SC. It is possible that in the current study, the IPM-induced reduced permeant pathlength diffusivity, changed SC lipid polarity and SC dehydration, may have had additive effects which enhanced the observed 2naphthol SC uptake, whilst at the same time reducing its pathlength diffusivity and hence overall flux. These processes seemed to augment 2-naphthol membrane holding capacity and could lead to the generation a permeant reservoir within corneocytes as previously observed with mercuric chloride (3).

\section{Microscopy}

MPLSM has been used to image visible light excitable fluorescent probes under passive $(28,55)$ and iontophoretic conditions $(36)$ in human skin. Yu et al. $(28,55)$ observed increased corneocyte uptake of sulphorhodamine B (hydrophilic), relative to a structurally related lipophilic probe, in the presence of oleic acid when applied in $50 \%$ EtOH/PBS. However, these probes had relatively large molecular weights $(\mathrm{MW}>550 \mathrm{Da})$ and contrasting lipophilicities but were applied at a finite dose with different thermodynamic activities.

The solubility of 2-naphthol in water is approximately 100 times less than $50 \%$ EtOH/water (Table 1) and this, in combination with partitioning effects, may be responsible for the markedly lower $R_{m}$ (Figure 2) and more uniform intercellular fluorescence distribution from water (Figure 4A) in comparison with the $50 \%$ EtOH/water vehicle (Figure 4G). The predominant partitioning of 2-naphthol in SC for all vehicles appears to be in the intercellular domain, although, there was some evidence of corneocyte uptake (Figure 4). Of particular note was the more ubiquitous distribution of 2-naphthol fluorescence in the SC from the IPM vehicle (figure 4J), compared with the other vehicles (figure 4A, D and $G$ ), suggested increased corneocyte uptake of the permeant. Another consideration is the fact that the lipid in 
the intercellular spaces will to a significant degree be replaced/diluted with ethanol/water, thus greatly increasing the capacity of intercellular spaces to dissolve 2-napthol. Therefore this does not imply that large amounts of 2-napthol are present in the corneocyte phase.

MPLSM images of 2-naphthol transport within the membrane, obtained in the current study, suggest that corneocyte uptake is vehicle dependent and is relatively low compared to solute concentration in the intercellular domain. A more definitive conclusion based on MPLSM image analysis was precluded by only semiquantitative agreement of MPLSM fluorescence data and transport partitioning data (Table 2). The semi-quantitative results for MPLSM fluorescence analysis are expected due to limitations of assessing MPLSM fluorescence intensity in the heterogeneous SC and vastly different properties of vehicles used. The contrasting vehicle properties could cause varying degrees of chemical quenching of 2-naphthol fluorescence in different media. It could be concluded though that the images were qualitatively consistent with the conclusions drawn from our transport data $\left(\mathrm{J}_{\max }\right.$ and $R_{m}$ ) and that combination of findings from the two methods significantly enhances interpretation of pharmacokinetic data.

\section{Conclusions}

The $J_{\max }$ and $R_{m}$ of 2-naphthol through human epidermis was highly vehicle dependent, with the $50 \% \mathrm{EtOH} /$ water vehicle showing significantly greater $\mathrm{J}_{\max }$ and $R_{m}$ values compared to $100 \%$ water, $20 \%$ PG/water and $100 \%$ IPM vehicles. Large differences in saturated solubility of 2-naphthol in each vehicle were responsible for the differences between the observed $k_{p}$ values, therefore analysing $J_{\max }$ was considered the best method of comparing the effects of different vehicles. The lower membrane penetration and higher membrane retention of 2-naphthol from IPM indicated that this vehicle may have applications for topical agents where stratum corneum retention and minimal systemic absorption are desirable.

MPLSM imaging of 2-naphthol indicated that partitioning was mainly into intercellular domain but accompanied by a vehicle dependent corneocyte uptake. This study illustrated that an integrated evaluation using MPLSM imaging and in vitro 
penetration and epidermal retention may enhance the understanding of epidermal solute transport.

\section{Acknowledgements}

We gratefully acknowledge the National Health \& Medical Research Council of Australia (NH\&MRC), Pfizer Inc, and the PAH Research Foundation for their financial support of this work. Human skin was obtained with the kind assistance of Dr Peter Catt, Nanette Rentoult and Juris Melgar. The authors are thankful to Dr Paul Masci and Miss Sheung-Yi Yuen for their practical and technical assistance. We are grateful to Dr Cameron Hayden for the helpful discussion of this manuscript.

\section{References}

1. P. M. Elias. Epidermal lipids, barrier function, and desquamation. J Invest Dermatol 80 Suppl: 44s-49s (1983).

2. R. J. Scheupleinand I. H. Blank. Permeability of the skin. Physiol Rev 51: 70247 (1971).

3. H. Bodde, I. Vandenbrink, H. Koerten, and F. Dehaan. Visualization of in vitro percutaneous penetration of mercuric- chloride - transport through intercellular space versus cellular uptake through desmosomes. . Journal of Controlled Release 15: 227-236 (1991).

4. J. Bouwstra, M. Devries, G. Gooris, W. Bras, J. Brussee, and M. Ponec. Thermodynamic and structural aspects of the skin barrier. . Journal of Controlled Release 15: 209-220 (1991).

5. R. R. Warner, K. J. Stone, and Y. L. Boissy. Hydration disrupts human stratum corneum ultrastructure. J Invest Dermatol 120: 275-84 (2003).

6. M. S. Roberts, S. E. Cross, and M. A. Pellett. Skin Transport. In K. A. Walters (ed), Dermatological and transdermal formulations (K. A. Walters, ed), Marcel Dekker, New York, 2002, pp. 89--195.

7. A. Rougier, C. Lotte, P. Corcuff, and H. I. Maibach. Relationship Between Skin Permeability and Corneocyte Size According to Anatomic Site, Age, and Sex In Man. Journal Of the Society Of Cosmetic Chemists 39: 15-26 (1988).

8. R. O. Pottsand M. L. Francoeur. The influence of stratum corneum morphology on water permeability. J Invest Dermatol 96: 495-9 (1991).

9. T. M. Sweeny and D. T. Downing. The Role of Lipids in the Epidermal Barrier to Water Diffusion. Journal of Investigative Dermatology 55: 135-139 (1970).

10. K. Abrams, J. D. Harvell, D. Shriner, P. Wertz, H. Maibach, H. I. Maibach, and S. J. Rehfeld. Effect of organic-solvents on in-vitro human skin water barrier function. Journal of Investigative Dermatology 101: 609-613 (1993).

11. T. F. Wang, G. B. Kasting, and J. M. Nitsche. A multiphase microscopic diffusion model for stratum corneum permeability. I. Formulation, solution, and 
illustrative results for representative compounds. J Pharm Sci 95: 620-48 (2006).

12. A. Rolland, N. Wagner, A. Chatelus, B. Shroot, and H. Schaefer. Site-specific drug-delivery to pilosebaceous structures using polymeric microspheres. Pharmaceutical Research 10: 1738-1744 (1993).

13. A. C. Lauer, L. M. Lieb, C. Ramachandran, G. L. Flynn, and N. D. Weiner. Transfollicular drug-delivery. Pharmaceutical Research 12: 179-186 (1995).

14. K. Higaki, C. Amnuaikit, and T. Kimura. Strategies for overcoming the stratum corneum. Am. J. Drug Deliv. 1: 187-214 (2003).

15. S. Gupta, A. Domashenko, and G. Cotsarelis. The hair follicle as a target for gene therapy. Eur J Dermatol 11: 353-6 (2001).

16. G. K. Menonand P. M. Elias. Morphologic basis for a pore-pathway in mammalian stratum corneum. Skin Pharmacol 10: 235-46 (1997).

17. M. K. Nemanicand P. M. Elias. A Novel Cytochemical Technique for Visualisation of Permeability Pathways in Mammalian Stratum Corneum. Journal of Histochemistry Cytochemistry 28: 573-578 (1980).

18. P. J. Caspers, A. C. Williams, E. A. Carter, H. G. Edwards, B. W. Barry, H. A. Bruining, and G. J. Puppels. Monitoring the penetration enhancer dimethyl sulfoxide in human stratum corneum in vivo by confocal Raman spectroscopy. Pharm Res 19: 1577-80 (2002).

19. G. Cevc, A. Schatzlein, and H. Richardsen. Ultradeformable lipid vesicles can penetrate the skin and other semi-permeable barriers unfragmented. Evidence from double label CLSM experiments and direct size measurements. Biochim Biophys Acta 1564: 21-30 (2002).

20. Y. Y. Gramsand J. A. Bouwstra. Penetration and distribution of three lipophilic probes in vitro in human skin focusing on the hair follicle. Journal of Controlled Release 83 253-62 (2002).

21. M. R. Prausnitz, J. A. Gimm, R. H. Guy, R. Langer, J. C. Weaver, and C. Cullander. Imaging regions of transport across human stratum corneum during high-voltage and low-voltage exposures. J Pharm Sci 85: 1363-70 (1996).

22. E. Touitou, N. Dayan, L. Bergelson, B. Godin, and M. Eliaz. Ethosomes - novel vesicular carriers for enhanced delivery: characterization and skin penetration properties. Journal of Controlled Release 65: 403-18 (2000).

23. N. G. Turnerand R. H. Guy. Iontophoretic transport pathways: dependence on penetrant physicochemical properties. J Pharm Sci 86: 1385-9 (1997).

24. M. E. van Kuijk-Meuwissen, H. E. Junginger, and J. A. Bouwstra. Interactions between liposomes and human skin in vitro, a confocal laser scanning microscopy study. Biochim Biophys Acta 1371: 31-9 (1998).

25. H. C. Gerritsenand C. J. De Grauw. Imaging of optically thick specimen using two-photon excitation microscopy. Microsc Res Tech 47: 206-9 (1999).

26. B. R. Masters, P. T. So, and E. Gratton. Multiphoton excitation fluorescence microscopy and spectroscopy of in vivo human skin. Biophys J 72: 2405-12 (1997).

27. B. R. Masters, P. T. So, and E. Gratton. Multiphoton excitation microscopy of in vivo human skin. Functional and morphological optical biopsy based on three-dimensional imaging, lifetime measurements and fluorescence spectroscopy. Ann N Y Acad Sci 838: 58-67 (1998).

28. B. Yu, C. Y. Dong, P. T. So, D. Blankschtein, and R. Langer. In vitro visualization and quantification of oleic acid induced changes in transdermal 
transport using two-photon fluorescence microscopy. J Invest Dermatol 117: 16-25. (2001).

29. E. Christophersand A. M. Kligman. Preparation of isolated sheets of human stratum corneum. Arch. Derm. 88: $702-704$ (1963).

30. J. P. Skelly, V. P. Shah, H. I. Maibach, R. H. Guy, R. C. Wester, G. L. Flynn, and A. Yacobi. FDA and AAPS report of the workshop on principles and practices of in-vitro percutaneous penetrationstudies: Relevance to bioavailability and bioequivalence. Pharmaceutical Research 4: 265-267 (1987).

31. J. Thomsonand D. A. Burns. LSC Sample Preparation by Solubilization, Packard Life Sciences manual, 2005.

32. S. E. Cross, W. J. Pugh, J. Hadgraft, and M. S. Roberts. Probing the effect of vehicles on topical delivery: understanding the basic relationship between solvent and solute penetration using silicone membranes. Pharm Res 18: 9991005. (2001).

33. C. Rosado, S. E. Cross, W. J. Pugh, M. S. Roberts, and J. Hadgraft. Effect of vehicle pretreatment on the flux, retention, and diffusion of topically applied penetrants in vitro. Pharm Res 20: 1502-7 (2003).

34. M. S. Roberts, R. A. Anderson, and J. Swarbrick. Permeability of human epidermis to phenolic compounds. J Pharm Pharmacol 29: 677--683 (1977).

35. C. G. J. Hayden. Sunscreens: Implications of their skin permeation, Department of Medicine, Vol. PhD, University of Queensland, Brisbane, 2002.

36. B. S. Grewal, A. Naik, W. J. Irwin, G. Gooris, C. J. de Grauw, H. G. Gerritsen, and J. A. Bouwstra. Transdermal macromolecular delivery: real-time visualization of iontophoretic and chemically enhanced transport using twophoton excitation microscopy. Pharm Res 17: 788-95 (2000).

37. B. M. Magnusson, Y. G. Anissimov, S. E. Cross, and M. S. Roberts. Molecular size as the main determinant of solute maximum flux across the skin. Journal of Investigative Dermatology 122: 993-9 (2004).

38. R. O. Potts and R. H. Guy. Predicting skin permeability. Pharm Res 9: 663-9 (1992).

39. D. A. W. Bucks, R. H. Guy, and H. I. Maibach. Percutaneous penetration and mass balance accountability - technique and implications for dermatology. Journal of Toxicology Cutaneous and Ocular Toxicology 8: 439-451 (1989).

40. T. Yano, A. Nakagawa, M. Tsuji, and K. Noda. Skin permeability of various non-steroidal anti-inflammatory drugs in man. Life Sci 39: 1043-50. (1986).

41. W. J. Pugh, M. S. Roberts, and J. Hadgraft. Epidermal permeability Penetrant structure relationships .3. The effect of hydrogen bonding interactions and molecular size on diffusion across the stratum corneum. International Journal Of Pharmaceutics 138: 149-165 (1996).

42. B. Berner, G. C. Mazzenga, J. H. Otte, R.J. Steffens, R.H. Juang, C.D. Ebert. Ethanol: water mutually enhanced transdermal therapeutic system II: skin permeation of ethanol and nitroglycerin. J Pharm Sci. 78: 402-7 (1989).

43. D. Bommannan, R. O. Potts, and R. H. Guy. Examination of the effect of ethanol on human stratum-corneum in vivo using infrared-spectroscopy. Journal of Controlled Release 16: 299-304 (1991).

44. C. Y. Goatesand K. Knutson. Enhanced permeation of polar compounds through human epidermis. I. Permeability and membrane structural changes in the presence of short chain alcohols. Biochim Biophys Acta 1195: 169-79 (1994). 
45. H. K. Vaddi, P. C. Ho, Y. W. Chan, and S. Y. Chan. Oxide terpenes as human skin penetration enhancers of haloperidol from ethanol and propylene glycol and their modes of action on stratum corneum. Biol Pharm Bull 26: 220-8 (2003).

46. B. Berner, R.H. Juang, G.C. Mazzenga. Ethanol and water sorption into stratum corneum and model systems. J Pharm Sci.;78: 472-6 (1989).

47. G. K. Menon, S. H. Lee, and M. S. Roberts. Ultrastructural Effects of some solvents and vehicles on the stratum corneum and other skin components: evidence for an "extended mosaic-partitioning model of the skin barrier". In M. S. Roberts and K. A. Walters (eds), Dermal absorption and toxicity assessment, Vol. 91 (M. S. Robertsand K. A. Walters, eds), Marcel Dekker, New York, 1998, pp. 727--751.

48. B. W. Barry. Lipid-protein partitioning theory of skin penetration enhancement. J Controlled Release 15: 237-248 (1991).

49. S. Tata, N. Weiner, and G. Flynn. Relative influence of ethanol and propylene glycol cosolvents on deposition of minoxidil into the skin. J Pharm Sci 83: 1508-10 (1994).

50. P. J. Frosch, U. Pekar, and H. Enzmann. Contact allergy to propylene glycol. Do we use the appropriate test concentration? Dermatol Clin 8: 111-3 (1990).

51. B. Mueller, Y. G. Anissimov, and M. S. Roberts. Unexpected Clobetasol Propionate Profile in Human Stratum Corneum after Topical Application In Vitro. Pharm Res 20: 1835-1837 (2003).

52. I. Brinkmannand C. C. Muller-Goymann. Role of isopropyl myristate, isopropyl alcohol and a combination of both in hydrocortisone permeation across the human stratum corneum. Skin Pharmacol Appl Skin Physiol 16: 393-404 (2003).

53. C. S. Leopold and B. C. Lippold. An attempt to clarify the mechanism of the penetration enhancing effects of lipophilic vehicles with differential scanning calorimetry (DSC). J Pharm Pharmacol 47: 276-81 (1995).

54. C. S. Leopold and B. C. Lippold. Enhancing effects of lipophilic vehicles on skin penetration of methyl nicotinate in vivo. J Pharm Sci 84: 195-8 (1995).

55. B. Yu, K. H. Kim, P. T. So, D. Blankschtein, and R. Langer. Visualization of oleic acid-induced transdermal diffusion pathways using two-photon fluorescence microscopy. J Invest Dermatol 120: 448-55 (2003). 